\title{
An Integrated Strategy for Implementation of Dried Blood Spots in Clinical Development Programs
}

\author{
Prajakti A. Kothare, ${ }^{1,2}$ Kevin P. Bateman, ${ }^{1,2}$ Marissa Dockendorf, ${ }^{1}$ Julie Stone, ${ }^{1}$ Yang Xu, ${ }^{1}$ \\ Eric Woolf, ${ }^{1}$ and Lisa A. Shipley ${ }^{1}$
}

Received 1 September 2015; accepted 11 December 2015; published online 8 February 2016

\begin{abstract}
Dried blood spot (DBS) sample collection has gained increased interest across the pharmaceutical industry as a potential alternative to plasma for pharmacokinetic (PK) evaluations. However, regulatory guidelines and examples of late-stage clinical trial applications in the literature are lacking. This paper communicates Merck's strategy for the implementation of DBS exemplified by experience on a late-stage program (MK-8931). In this program, DBS was proposed as the sole matrix for phase 3 studies to decrease logistical burden in an aging target patient population (Alzheimer's disease). In vitro and bioanalytical tests demonstrated initial method feasibility and suitability for further evaluations in the clinic. An in vivo dataset was developed initially in healthy subjects (phase 1 study) and then in patients (phase 2/3 study) to establish a quantitative relationship between the blood and plasma concentrations (bridging dataset) using descriptive and population PK analyses. This allowed for PK conclusions to be seamlessly drawn across the clinical program without impact from the choice of matrix. This integrated information package (in vitro, bioanalytical and clinical) was presented to major regulatory agencies (FDA and EMA) for regulatory input. Based on this package, regulatory concurrence was gained on accepting DBS as the sole matrix in late-stage clinical trials.
\end{abstract}

KEY WORDS: bridging; dried blood spots; MK-8931; population PK.

\section{INTRODUCTION}

Since its original application for the detection of phenylketonuria in neonates half a century ago, dried blood spots (DBS) have gained popularity as a screening tool for various diagnostic tests including metabolic disorders, therapeutic drug monitoring, and HIV infection in neonates (1-3). The pharmaceutical industry and regulators have continued to explore the potential of DBS as a viable alternate matrix for pharmacokinetic analyses (4-6). DBS was initially evaluated at Merck in 2001 for discovery stage PK studies and subsequently implemented for the pediatric development program of an anti-HIV compound in 2009. Since then, a number of compounds have been evaluated for initial in vitro and bioanalytical feasibility to implement DBS. A subset of these has progressed to implementation of DBS in clinical trials, including late-stage clinical trials. Strategically, Merck has chosen to primarily focus the application of DBS towards late-stage patient studies (phase 2 and/or 3) where it has the potential to render greater impact. In our opinion, the value proposition for DBS from a clinical perspective is as follows:

\footnotetext{
${ }^{1}$ Pharmacokinetics, Pharmacodynamics and Drug Metabolism, Merck \& Co., Inc., 770 Sumneytown Pike, West Point, Pennsylvania 19486, USA.

${ }^{2}$ To whom correspondence should be addressed. (e-mail: kevin_bateman@merck.com; prajakti_kothare@merck.com)
}

(a) To add flexibility in the collection of PK data in phases 2 and 3 studies: Typically, sparse PK samples in phases 2 and 3 studies are constrained to limited time windows during a clinic visit. DBS, particularly in an out-patient setting, expands the window for access to such data. This may be especially beneficial for drugs with long half-lives or long acting formulations to evaluate steady-state or time for washout, or where clinical endpoints are collected by patientcompleted diaries or are episodic (e.g., migraine, asthma, or erectile dysfunction trials). However, sampling methods in an out-patient setting need to mature further to gain adequate precision for pharmacokinetic modeling.

(b) Decreased patient burden (blood volume) in vulnerable populations: The smaller sample volumes typically associated with DBS (three spots of $\sim 20-40 \mu \mathrm{L}$ each) vs. typical plasma samples $(\sim 200-1000 \mu \mathrm{L})$ are clinically attractive for vulnerable populations where blood volumes are a clinical or ethical concern (e.g., younger pediatric or elderly populations). However, DBS should not be considered an automatic choice for studies in such populations. Equal consideration should be given to liquid microsampling approaches.

(c) Improved logistical feasibility: DBS sampling offers a number of logistical advantages (e.g., ambient temperature storage or shipping, no need for specialized equipment such as refrigerated centrifuges and 
simplified sample preparation) that may reduce operational burden associated with PK sampling in larger multi-center patient trials and lead to potential cost savings. A reduced operational burden may encourage greater participation of clinical sites for PK evaluation in phases 2 or 3 trials, and thereby enrich the database for characterization of the population pharmacokinetics and exposure-response relationships. Prior to implementing ambient shipping for DBS, the extended stability of DBS samples should be established to ensure integrity of the samples.

DBS has been used extensively for the measurement of endogenous biomarkers of disease $(7,8)$. Merck is exploring the use of DBS for clinical laboratory tests and biomarkers. Continued development of these approaches and coordination with PK measurements is anticipated to further enhance the value proposition for DBS in clinical trials.

Despite the upside potential of DBS, companies and regulators have wrestled with its utility and regulatory expectations compared to traditional matrices. Recently, the European Bioanalysis Forum (EBF) and IQ have published position papers reflecting a range of opinions on DBS $(9,10)$. However, industry regulatory consensus positions and regulatory guidelines for DBS in clinical development programs have been slow to emerge. Further, with few exceptions, the literature has focused on technological challenges and bioanalytical considerations. Rowland and Shepard provide an overview of the requirements for the interpretation of DBS data in development and addressed some of the regulatory considerations that would pave the way to gaining acceptance for DBS (11). A theoretical assessment of pharmacokinetic considerations in the interpretation of DBS data has been published by Emmons and Rowland (12). However, the literature lacks in-depth guidance on pharmacokinetic analyses, modeling, or late-stage clinical considerations, especially when integrating across a clinical program with studies that include both plasma and DBS sampling.

The use of DBS should be thoughtfully weighed on a case-by-case basis with consideration to pros/cons of its use relative to traditional matrices for a given program. Successful implementation hinges on prospective multi-disciplinary (e.g., Bioanalytical, pharmacokinetic-pharmacodynamic (PKPD), Clinical Pharmacology, regulatory) planning. As most clinical development programs employ plasma sampling for phase 1 studies, a robust in vivo bridging strategy should be developed that allows quantitative inter-conversion of pharmacokinetic information between matrices. This allows pharmacokinetic conclusions to be drawn across studies agnostic to matrix. The overarching PKPD objectives for the program (e.g., evaluation of intrinsic/extrinsic factors, exposure-response) should remain a core consideration and not be impacted by the choice of the bioanalytical matrix.

This paper presents Merck's strategy for the application of DBS in clinical programs which has been shaped by experience gained from implementation on clinical programs. This augments an earlier company position paper where initial experience, bioanalytical, and logistical considerations were communicated (6). Of note, this summary is intended to inform the scientific community of an emerging area of interest with limited industry or regulatory precedence and is not intended to represent a broader consensus position by regulators or industry. The strategy is composed of staged implementation steps where initial methodological feasibility is established through a series of in vitro and bioanalytical evaluations. DBS is introduced in a staged manner in the clinic whereby DBS samples are taken concurrently with plasma initially in a healthy subject study and then in patients. Descriptive and population PK analyses of these data are conducted in a "learn and confirm" paradigm. Regulatory feedback is sought on this comprehensive data package (Fig. 1). Implementation of this strategy is exemplified through experience gained from MK-8931, a clinical program where regulatory concurrence was gained for the acceptance of DBS as the sole PK collection matrix for late phase trials.

\section{CASE STUDY: MK-8931}

Strategic Rationale. MK-8931 is currently in late-stage development for Alzheimer's disease. DBS would allow for shipping at ambient temperature and render a number of other logistical advantages that could encourage clinical site participation and facilitate faster study enrollment for large multi-site clinical trials. Additionally, the reduced blood volume associated with DBS collection was anticipated to reduce patient burden. The goal of implementation was to pursue DBS as the sole matrix for late-stage clinical trials. The characterization of exposure-response relationships was considered an important element for dose justification. Thus, the bridging package needed to be robust to allow clinical studies to be pooled across the clinical program for population PK and exposure-response analyses in anticipation of regulatory submission and labeling.

\section{Initial Feasibility Assessment}

Initially, a set of in vitro and bioanalytical assessments were conducted to ascertain methodological feasibility prior to further evaluations in the clinic. These tests were conducted over a clinically relevant concentration range and required good communication between the bioanalytical and PKPD scientists.

In Vitro Tests. As detailed in the publication of Rowland and Emmons (12), plasma protein binding, blood-cell to unbound plasma concentration ratio $(\rho)$, and hematocrit are important in vitro determinants of the blood to plasma ratio and the suitability of DBS as a pharmacokinetic matrix. Emmons and Rowland hypothesize that compounds with a blood to plasma ratio ranging from 0.55 to 2 , and non-concentration-dependent unbound fraction (fu) and $\rho$ can be equally analyzed by blood or plasma $(5,12)$.

The average MK-8931 in vitro blood to plasma ratio was 1.22 at $1 \mu \mathrm{M}$ based on radiolabelled experiments. The blood to plasma ratio was concentration independent in the range of 0.03 to $10 \mu \mathrm{M}$ (bracketing the clinical concentration range). MK-8931 was modestly plasma protein bound ( $\sim 65 \%$ in human plasma) and not concentration dependent in the range of 0.03 to $1 \mu \mathrm{m}$. The in vitro tests showed that DBS or plasma would be equally valid as a matrix for MK8931 PK assessments. 


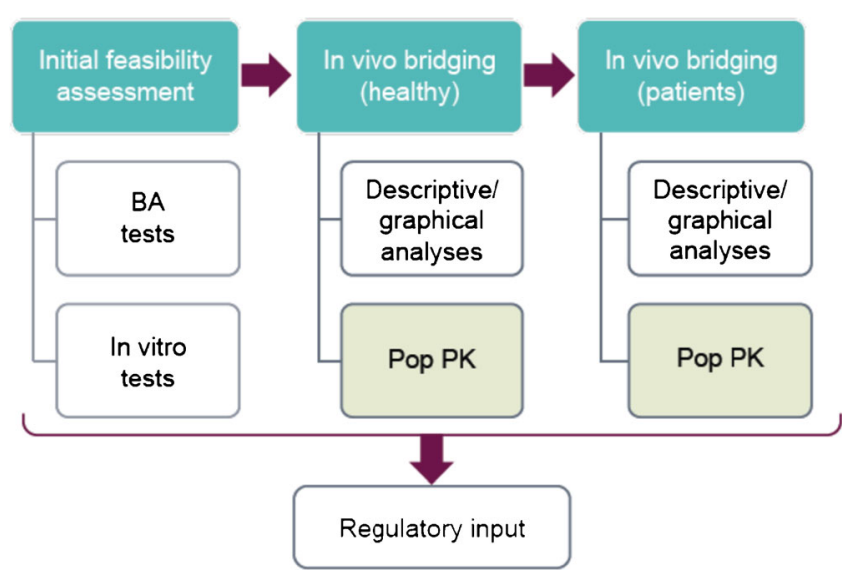

Fig. 1. Components of the integrated DBS strategy

Bioanalytical Tests. Prior disseminations summarize Merck's strategy for evaluating bioanalytical feasibility $(6,13)$. These tests encompass assay sensitivity and range, DBS card type and extraction methods, the impact of hematocrit on the bioanalytical method over a wide range, impact of spot volume/homogeneity, stability (including relevant metabolites) at extremes of temperate and relative humidity (potentially encountered during shipping), and any considerations specific to the quantitation of the molecule (e.g., concentration-dependent binding to target and/or plasma). There are currently no established regulatory guidances for bioanalytical method development or validation specific to DBS. Hence, following a decision to pursue DBS, regulatory bioanalytical guidelines, internal SOPs, and industry best practices for plasma assays are applied for bioanalytical validation.

For MK-8931, venous blood was drawn into a single EDTA collection vial and a small quantity spotted onto a DBS card; the remaining blood sample was centrifuged to extract plasma. MK-8931 concentrations were analyzed in both the DBS and the plasma samples. The DBS analytical method was based on either direct extraction or direct extraction followed by liquid-liquid extraction of MK-8931 from human dried blood spots on DMPK-A cards. The analyte from DBS samples and its stable isotope labeled internal standard contained in the extraction solvent were analyzed by HPLC-MS/MS. The lower limit of quantitation (LLOQ) was $1 \mathrm{ng} / \mathrm{mL}$ with a 3-mm punch size and was deemed adequate for clinical trials. Hematocrit was shown not to have an analytically relevant effect on MK-8931 concentration measurements in the range of 19.5 to $86.0 \%$. Following the receipt of clinical study samples, incurred sample reanalysis (ISR) was completed to further validate the reproducibility of the assay methodology. Assay validation and clinical performance are summarized in Tables I and II. Bland-Altman plots comparing DBS and plasma concentrations showed a lack of bias between the methods (Fig. 2). The DBS analytical method was considered to have robust performance for use in a clinical setting.

In other instances, bioanalytical evaluations have ruled out DBS as a possible matrix. For example, one compound exhibited high levels of a circulating glucuronide metabolite and stability testing of DBS samples containing the glucuronide showed that it back-converted to parent compound at both room temperature and low temperature with acidification. This would confound the accurate measurement of the parent compound. Therefore, traditional plasma sampling with acidification to stabilize the metabolite was considered a more viable option, and DBS was not further pursued. In other instances, DBS has been successfully evaluated in early clinical studies, but the program failed to advance, and therefore, no further DBS data were collected (13).

\section{In Vivo Bridging}

\section{General Considerations}

As most clinical development programs are initiated with, and likely to retain phase 1 studies with plasma assays, a quantitative in vivo relationship between blood and plasma concentrations is established through "bridging studies." These typically encompass a staged evaluation commencing in a healthy subject study and then proceeding to a patient study. In each study, DBS and plasma samples are taken concurrently at various PK sampling time points that span a therapeutically relevant concentration range. Depending on whether the proposed future clinical trial (after bridging is established) would continue blood collection through venipuncture or in an out-patient setting, DBS may be collected via venipuncture in the clinic or through finger sticks. As sample collection has a direct impact on the quality of DBS assessments, Merck has developed extensive training material

Table I. Assay Validation Performance Summary for MK-8931

\begin{tabular}{|c|c|c|c|c|}
\hline Assessment & Samples/Conditions Assessed & $N$ & Mean Accuracy (\%) & $\begin{array}{l}\text { Mean Precision } \\
\text { (Coefficient of } \\
\text { Variation \%) }\end{array}$ \\
\hline Regression model analysis & $\begin{array}{l}\text { Replicate standard curves } \\
\quad\left(\text { linear, } 1 / \mathrm{x}^{2}\right)\end{array}$ & 5 & $92.0-105$ & $3.7-7.3$ \\
\hline Intra-run accuracy and precision at the LLOQ & 3 core runs & 5 in each run & $101-111$ & $9.2-18.9$ \\
\hline $\begin{array}{l}\text { Intra-run accuracy and precision at low, mid, } \\
\text { and high QC }\end{array}$ & 3 core runs & 5 in each run & $99.4-109$ & $2.4-7.3$ \\
\hline $\begin{array}{l}\text { Inter-run accuracy and precision at LLOQ, } \\
\text { low, mid, and high QC }\end{array}$ & Mean of 3 core runs in 3 days & 3 & 101-107 & $0.8-4.9$ \\
\hline
\end{tabular}


Table II. Assay Clinical Study Performance Summary for MK-8931

\begin{tabular}{|c|c|c|c|c|c|c|c|c|}
\hline \multirow[b]{2}{*}{ Clinical Protocol } & \multicolumn{4}{|c|}{ Mean Accuracy (\%) } & \multicolumn{4}{|c|}{ Mean Precision (Coefficient of Variation \%) } \\
\hline & $N$ & QC L & QC M & QC H & $N$ & QC L & QC M & QC H \\
\hline PNOA & 6 & 93.7 & 103 & 95.9 & 6 & 14.6 & 5.80 & 3.84 \\
\hline PNOB & 40 & 99.9 & 102 & 101 & 40 & 8.44 & 2.87 & 5.21 \\
\hline
\end{tabular}

(including videos and educational materials) for sites for guidance on sample handling, and shipping. The participation of PKPD and/or BA scientists in start-up meetings is encouraged to ensure participating sites are appropriately educated on practical considerations of sample collection and handling.

For the healthy subject evaluation, an existing clinical pharmacology study (e.g., multiple ascending dose, control arm of a DDI, or special population study) should be leveraged. No a priori sample size is recommended; a typically sized clinical pharmacology study (e.g., 12-16 subjects) is sufficient. Subsequently, bridging data should be collected from an early patient study (e.g., phase 1b or POC).

Merck utilizes a "weight of evidence" approach that includes a series of graphical/descriptive evaluations and with equal or greater weightage given to population pharmacokinetic analyses. These analyses should follow a learn and confirm paradigm. Analyses should commence with the healthy subject study in an exploratory or "learning" mode and be confirmed in the patient study. Based on input provided for MK-8931 during the EMA oral hearing, an external qualification of the blood-plasma relationship is highly desirable. Establishing and qualifying this relationship enables pharmacometric analyses of concentration data across studies in a clinical program in support of the submission package and labeling.

\section{Healthy Study}

For MK-8931, DBS was initially included along with plasma concentrations in healthy subjects in a clinical pharmacology study (12 subjects contributing 11 samples each). DBS samples were collected via venipuncture in the clinic. A number of graphical and descriptive analyses were conducted. Concurrently drawn DBS and plasma concentrations were plotted to explore data trends (Fig. 3a). The blood to plasma slope $(95 \% \mathrm{CI})$ estimated by regression was 1.29

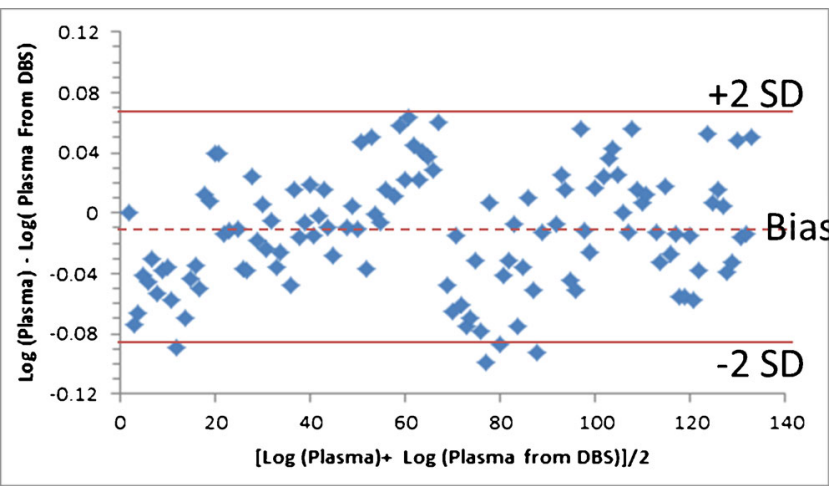

Fig. 2. Bland-Altman plot comparing plasma and DBS concentrations for pharmacokinetic samples from the phase 1 study for MK-8931
(1.27, 1.31), which was in close agreement with the in vitro estimated blood to plasma (B:P) ratio of 1.22. Of note, while regression fits may be applied to quantify the relationship, a prospective $R^{2}$ cutoff should not be applied as a go/no go criterion. Further, non-linear trends should not be inferred as a lack of utility of DBS. Observed mean (Fig. 3b) and individual (Fig. 3c) concentrations were plotted by nominal time. The plots include MK-8931 concentration data derived from plasma, DBS, and "DBS-predicted plasma concentrations" (DBS concentration divided by the slope from regression fit of 1.29). The measured and DBS converted plasma concentrations were generally comparable and followed similar trends over time. These graphical and descriptive analyses suggested that the in vivo B:P relationship was well characterized in healthy subjects.

In Merck's experience, the aforementioned graphical plots have been well received by regulators. Additionally, a mixed-effects modeling approach could be applied to evaluate the blood and plasma concentration data. In this approach, time-matched DBS and plasma concentration data pairs are fitted to a mixed-effects model (e.g., $\ln (\mathrm{DBS})=$ slope $^{*} \ln ($ plasma $\left.)\right)$ in a program such as NONMEM. Inter- and intra-individual variability terms as well as the influence of covariates such as hematocrit may be evaluated using this approach.

Equal or greater weightage was applied to population PK as a critical element of the overall bridging strategy. Implementation followed a learn and confirm paradigm as shown in Fig. 4. MK-8931, DBS, and plasma concentration data from the phase 1 study were used to update an existing plasma population pharmacokinetic model. Blood concentrations were modeled as a separate compartment with an estimated population "slope" that related blood and plasma concentrations (Fig. 5). Separate residual error terms were applied for blood and plasma. Parameter estimates and errors were similar between a model that included plasma data alone and one that included plasma and DBS and were consistent with historical knowledge of the compound (Table III). The population slope ( $\%$ relative standard error) estimated from the model was $1.27(4.61 \%)$ and similar to that obtained from the regression analyses and the in vitro blood to plasma ratio.

Fig. 3. a Correlation of blood and plasma concentration data from a phase 1 bridging study of MK-8931. b Mean plasma and blood concentration-time data from a phase 1 bridging study of MK-8931. Note: DBS-predicted plasma concentrations were calculated as measured DBS divided by 1.29, the slope of the DBS-plasma linear regression line. c Plasma and blood concentration-time data for individual subjects from a phase 1 bridging study of MK-8931. DBSpredicted plasma concentrations were calculated as measured DBS divided by 1.29 , the slope of the DBS-plasma linear regression line 
a
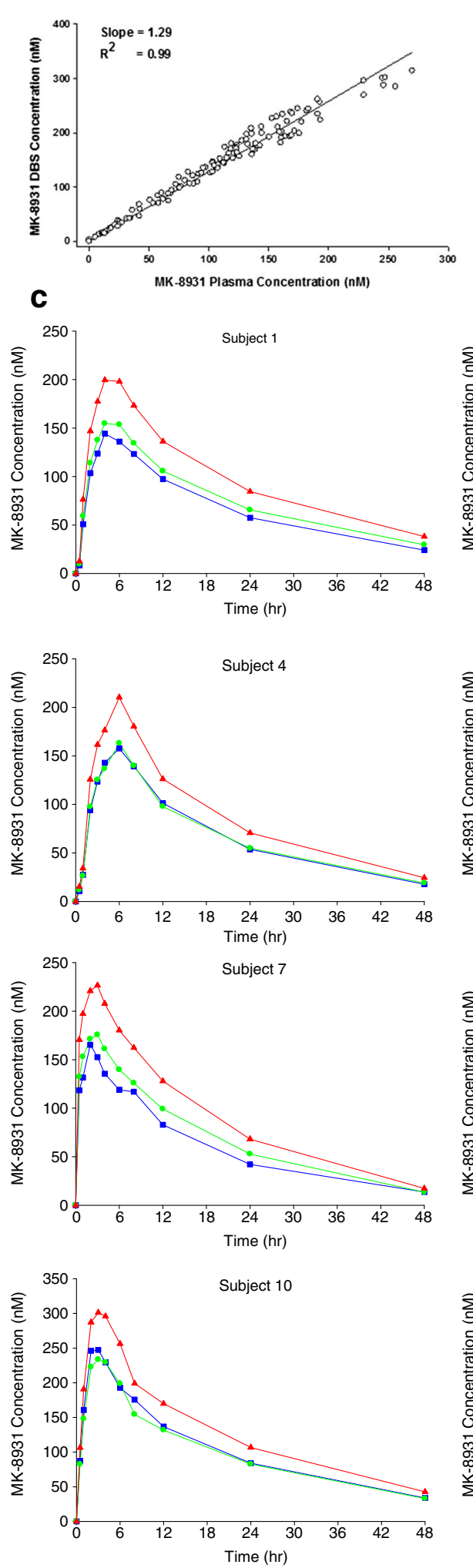

b
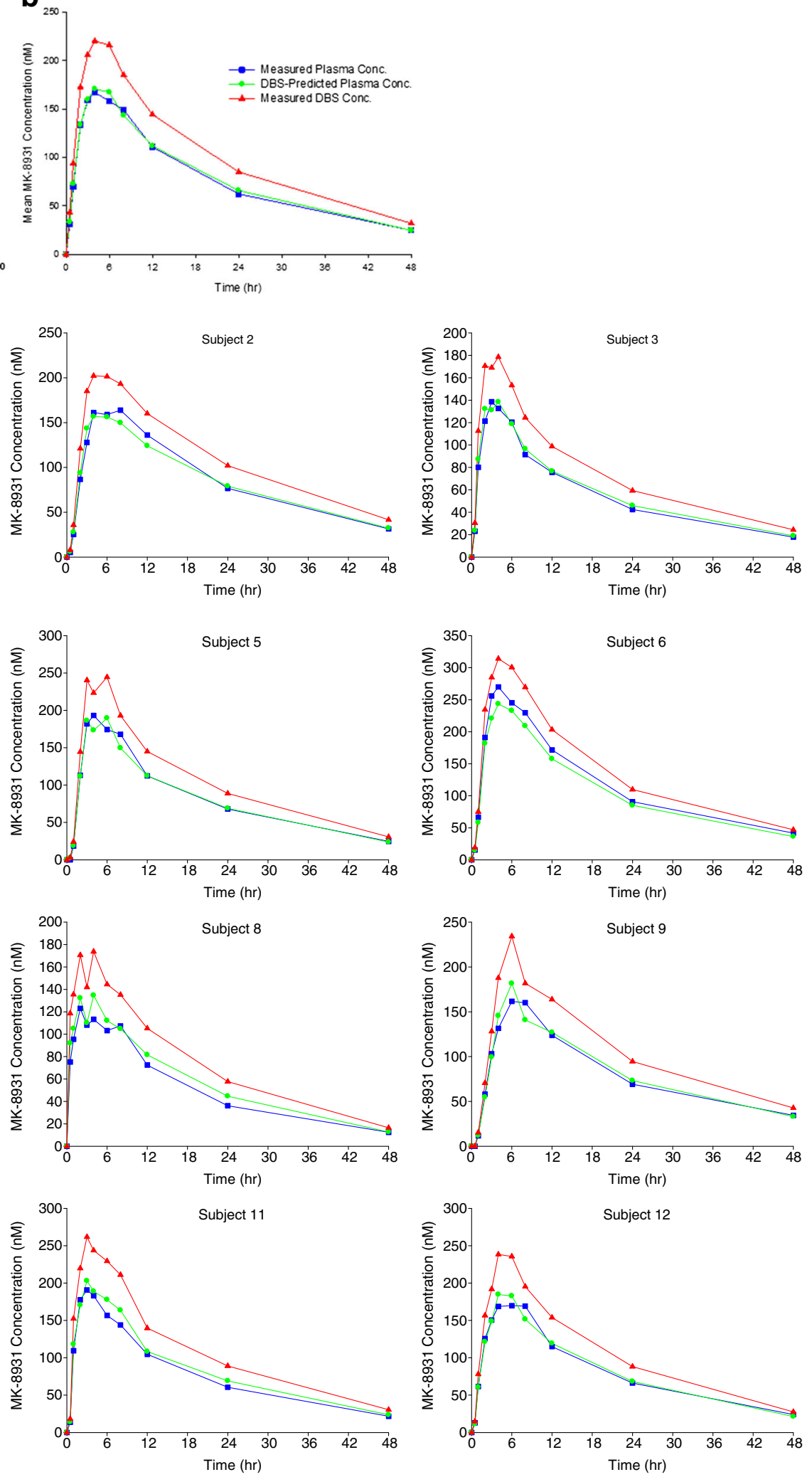

Measured Plasma Conc

DBS predicted Plasma Conc [Measured DBS/Slope]

Measured Blood Conc (DBS) 


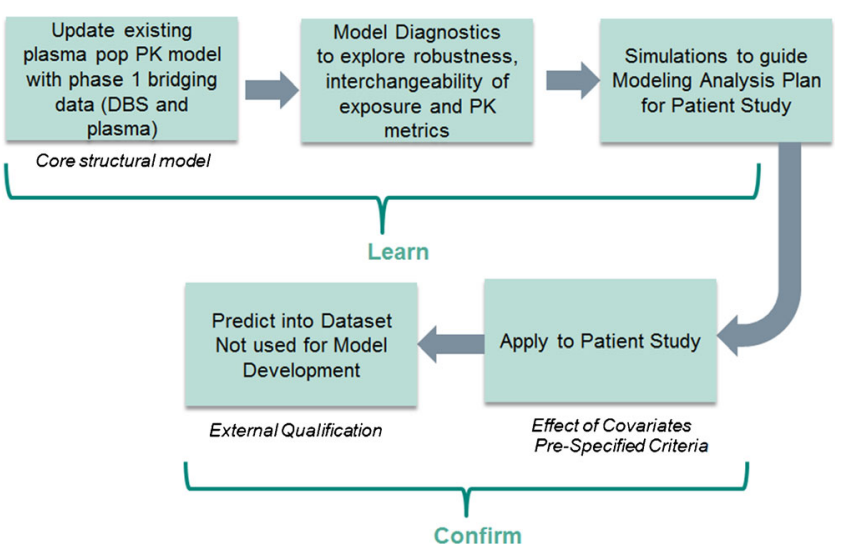

Fig. 4. Road map for application of population PK to establish a quantitative bridge between plasma and DBS concentrations

The impact of inter-individual variability on slope was explored and found not to be significant. Existing covariate relationships from the plasma model were applied to the plasma-DBS model; however, the impact of covariates on the slope was not evaluated at this stage. Random effects and residual errors for the parameters were within reasonable limits. Other key modeling parameters (e.g., clearance) were also well estimated which would be of relevance to support covariate analyses (a key objective for the phase $2 / 3$ population PK evaluations).

Modeling best practices and diagnostics were applied to explore the goodness of fit and the quantitative interchangeability of exposure metrics derived from the two matrices. Standard diagnostics and goodness of the fit plots such as visual predictive checks or CWRES (conditional weighted residuals) or IWRES (individual weighted residuals) vs. time plots differentiated by matrix were used to rule out the patterns of systematic bias or model mis-specification. Another useful diagnostic was to compare the individual post hoc plasma exposures from the plasma-DBS bridging study using (1) the plasma data from the bridging study and the plasma alone model and (2) the DBS data from the bridging study and the plasma + DBS model, using model-estimated slope to convert to a plasma exposure. DBS-based exposure estimates were similar and did not show any over- or under-prediction bias compared to the plasma-based estimates (Fig. 6).

Of note, while the blood to plasma relationship of MK8931 was linear, if the underlying relationship for a given compound is non-linear, a more complex parametric description could be developed. However, the increased

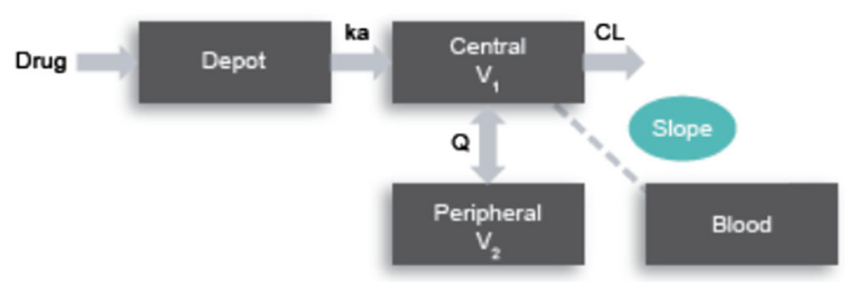

Fig. 5. A base population pharmacokinetic model structure that relates plasma and DBS concentration data by a population estimated slope. See Appendix for example NONMEM code parameterization would need to be balanced relative to the overall advantages conferred by DBS to the program.

\section{Patient Study}

Descriptive and population analyses in healthy subjects supported the continued utility of DBS. Therefore a subsequent bridging dataset was developed in patients to confirm this relationship in a more heterogeneous and relevant patient population. For MK-8931, this was obtained from an early cohort of patients in a late-phase clinical trial. Concurrent DBS and plasma samples by venipuncture in clinic were taken pre-dose and as three sparse PK samples over a 13week period. Additional DBS and plasma samples continued to be collected from the study based on advice received at an EMA oral hearing to develop an external qualification dataset.

Simulations using the phase 1 pop PK model were used to develop prospective go/no go decision criteria and included in the modeling analysis the plan for the patient study to support the decision of whether DBS could be used as the sole matrix for future phase 3 studies. The modeling analysis plan would evaluate (i) comparability of model-estimated slope to regression estimated (in vivo) and in vitro blood to plasma ratio, (ii) similarity and lack of bias in parameters and post hoc exposures derived from plasma and DBS converted to plasma, and (iii) similar central tendency of plasma predicted $v s$. DBS converted plasma predicted exposures. For MK-8931, two decision trees were included in the modeling analysis plan (Fig. 7). These decision trees represent program-specific criteria and need to be adjusted on a case-by-case basis. Based on discussions at the EMA oral hearing, the modeling plan was updated to include an external qualification step where the DBS-plasma population PK model (including the population slope term) would also be used to estimate exposures (based on DBS data alone) for an additional set of patients not utilized for the model development.

A similar set of descriptive and graphical analyses as those performed on data from healthy subjects was planned for data from the patients. Furthermore, the population PK model would be updated with patient-derived DBS data and assessed relative to the proposed modeling plan and decision trees. Additional patient data from this data, not included in model development, would be used for external qualification. In its totality, the modeling plan was designed to evaluate whether DBS could achieve the broader PK objectives of the clinical program, such as application for exposure-response modeling, with a similar degree of fidelity as plasma. These pharmacometric assessments (as well as the preceding strategic, in vitro and bioanalytical components) were a critical aspect of the package submitted to regulatory agencies for feedback on whether DBS would be suitable as the sole matrix for late-stage studies. For blinding considerations, results from the patient study are not included in the current dissemination. These will be the subject of a subsequent external communication upon unblinding of the study.

\section{Regulatory Input}

As clinical application of DBS is still emerging, regulatory guidance on DBS is yet to be established. Thus, 
Table III. Phase 1 MK-8931 Population PK Model Parameter Estimates (\% Residual Standard Error) for Relevant Parameters

\begin{tabular}{llll}
\hline & & Plasma-Only Model $^{a}$ & ${\text { Plasma }+ \text { DBS Model }^{b}}^{\text {Parameter }}$ \\
\cline { 3 - 4 } & Parameter Description & Estimate (\%RSE) & Estimate (\%RSE) \\
\hline Slope & DBS/plasma ratio & - & $1.27(4.61)$ \\
$\sigma^{2}$ plasma & Additive residual variability for plasma & $0.142(7)$ & $0.144(7.29)$ \\
$\sigma^{2}$ DBS & Additive residual variability for DBS & - & $0.186(34.7)$ \\
\hline
\end{tabular}

${ }^{a}$ Model developed using phase 1 plasma data

${ }^{b}$ Model developed using Phase 1 plasma data as well as DBS data from a healthy volunteer bridging study

feedback from key regulatory agencies is recommended in a timely manner for each program. The briefing package should present an integrated assessment of in vitro data, bioanalytical feasibility assessments, and in vivo bridging evaluations. The timing of such correspondence is program specific and should enable subsequent finalization of latestage clinical plans. We recommend that meeting requests specify input from pharmacometric and bioanalytical reviewers. The inclusion of external expert opinions as part of the submitted dossier may be considered. It is Merck's experience that comprehensive data packages that included the elements mentioned above enabled more productive regulatory interactions.

This approach was used for MK-8931. The focus of the interactions was to gain regulatory concurrence that the submitted package supported the use of DBS (collected in the clinic via venipuncture) as the sole method of PK sampling in ongoing and future MK-8931 late-stage clinical trials. For MK-8931, FDA interactions occurred at an earlier time frame when the DBS-associated pharmacometric package was not as well developed. The submitted package primarily relied on in vitro and BA feasibility assessments and descriptive/graphical analyses of phase 1 clinical data along with plans for the collection of plasma and DBS bridging data in patients, and the agency agreed that our proposal appeared reasonable. During correspondence, the agency recommended the use of the individual concentration time profiles as denoted in Fig. 3c. A comprehensive background document (inclusive of pharmacometric evaluations) was submitted for scientific advice to the CHMP/EMA. The agency requested an oral hearing as they indicated that this was their first regulatory experience of DBS. The key input received were the following:

- Merck has presented a comprehensive approach consisting of bioanalytical feasibility considerations and in vitro studies followed by an in vivo bridging program in both healthy and patient populations. Overall, this approach was considered robust to support the use of DBS as the sole source of PK

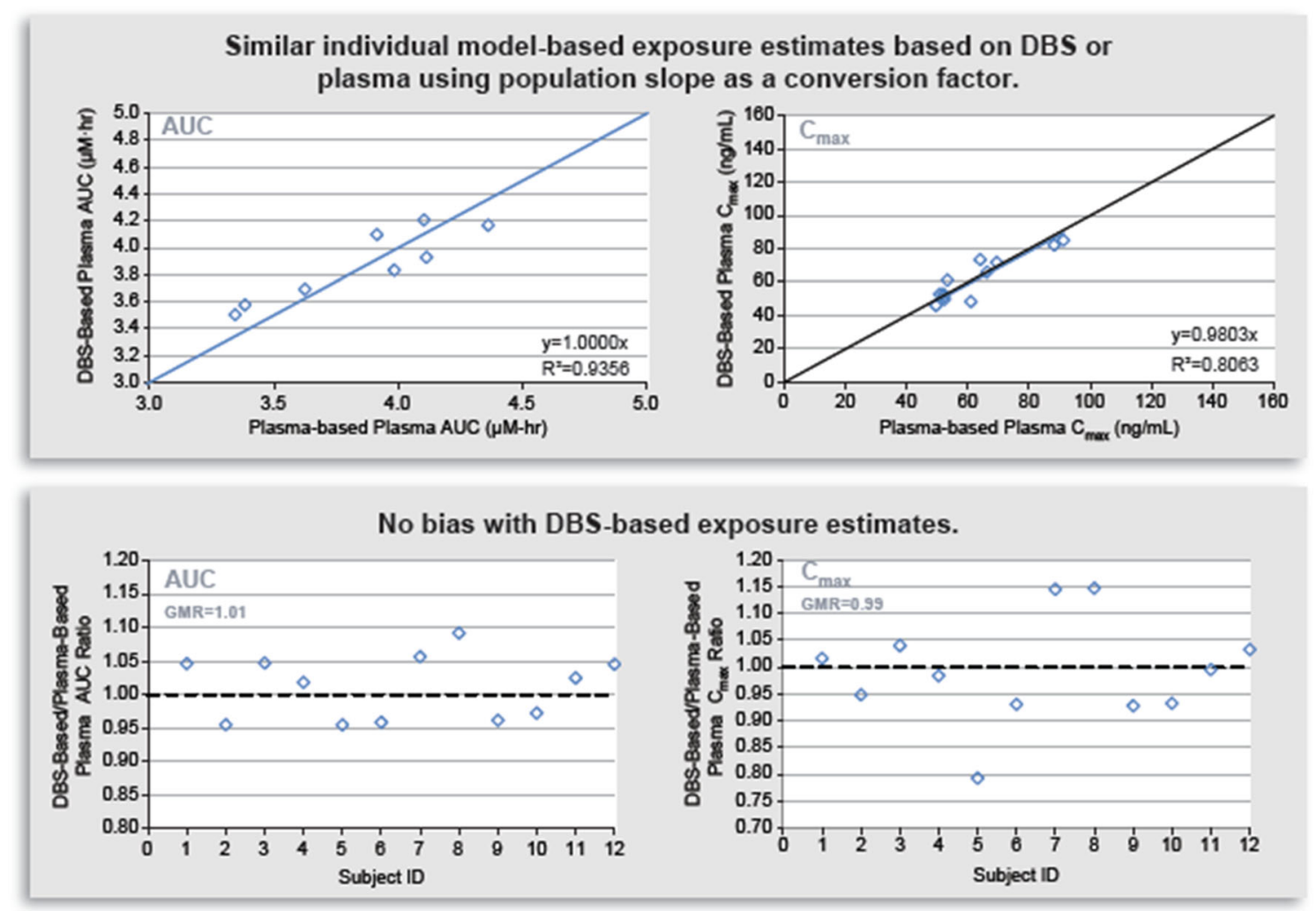

Fig. 6. Individual MK- 8931 model-predicted exposures using plasma alone data and model $v s$. from DBS concentration data converted to plasma using the model-estimated population slope 


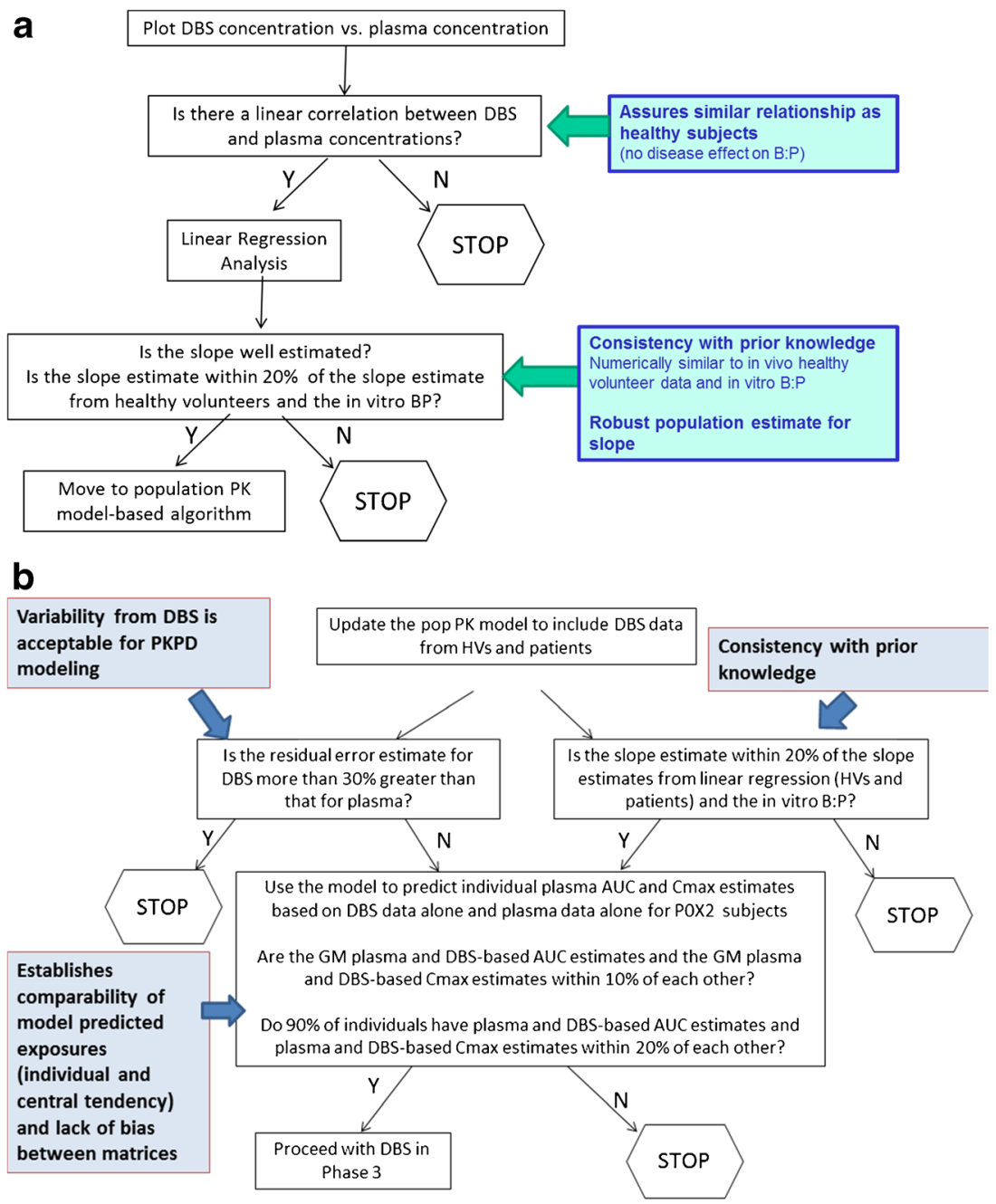

Fig. 7. a MK-8931 DBS-plasma decision tree no. 1 (linear regression analysis based on patient data only). b MK-8931 DBS-plasma decision tree no. 2 (population PK modelbased analysis based on healthy volunteer and patient data)

data for the remainder of the MK-8931 phase 3 program.

- While the broader strategy was endorsed, the agency cautioned that the implementation of DBS requires unique considerations which may not be readily translatable to other development programs.

- There was a strong focus on trying to understand the impact of inter-individual variability on the slope and to ensure that the blood to plasma relationship (i.e., slope) could be applied to describe populations not included in the modeling dataset. The agency recommended that external qualification of the slope be demonstrated to show its predictive value in a dataset not used for model development.

- Of note, while the agency acknowledged the potential future benefits of home sampling, they refrained from providing commentary as they considered it to be technology in the early stages of development.

As stated before, the dissemination of this regulatory interaction is intended to advance the field by sharing knowledge in an area with relatively little regulatory precedence or guidance and as such is not intended to reflect a broader regulatory position statement.

\section{DISCUSSION}

The strategy for using DBS in clinical programs at Merck has been developed over several years and has been a non-linear process. Merck arrived at its current state by looking at DBS holistically in the context of individual development programs, avoiding a one-size-fitsall approach. Early efforts mainly focused on analytical aspects, and it was only after a whole program approach was adopted, which the broader utility of DBS has started to be realized. Merck's strategy requires a prospective and multi-study approach to build the data sets to enable successful clinical implementation. As such, the alignment of all groups (analytical, pharmacokinetics, clinical, operations) involved is critical to the successful implementation of DBS. The input from regulatory agencies has been critical to the refinement of our strategy. The feedback on 
both the analytical and pharmacokinetic aspects demonstrated scientific insightfulness and curiosity, while embracing a forward-looking attitude. As with any new approach, Merck anticipates unexpected hurdles during the development and implementation stages. Learning from the large-scale implementation of DBS in these trials is likely to benefit the broader community and will be the subject of future disseminations.

\section{Future Perspective}

Merck believes that the strategies described above will help transform DBS from a niche bioanalytical technology to a practical clinical strategy that can be leveraged for population-based PKPD models suitable for regulatory submissions. Presentations of such data packages to regulators should build regulatory confidence in acceptance of DBS as a mainstream matrix and presumably influence future regulatory guidance. Merck believes that investing in novel methodologies such as these is an essential part of the solution to addressing rising drug development costs, while still meeting regulatory agencies expectations to demonstrate a robust understanding of the PKPD relationship of new therapeutic agents and demands by payers to reduce costs.

\section{ACKNOWLEDGMENTS}

The authors would like to thank PPDM staff and external collaborators Malcolm Rowland, David Jaworowicz (Cognigen), and Julie Passerell (Cognigen) for their valuable input over the years that has led to the development of a clinical strategy for the implementation of dried blood spots at Merck.

Open Access This article is distributed under the terms of the Creative Commons Attribution 4.0 International License (http://creativecommons.org/licenses/by/4.0/), which permits unrestricted use, distribution, and reproduction in any medium, provided you give appropriate credit to the original author(s) and the source, provide a link to the Creative Commons license, and indicate if changes were made.

\section{APPENDIX}

Population Analyses of DBS and Plasma Concentration Data: Excerpt from NONMEM Code within \$ERROR Block $\mathrm{Q} 1=0$

IF (CPT.EQ.2) Q1 = 1

IF (CPT.EQ.2) IPRED = LOG $(\mathrm{F})$

$\mathrm{Y} 1=\mathrm{IPRED}+\mathrm{EPS}(1)$
$\mathrm{Q} 2=0$

IF (CPT.EQ.3 .AND. F.GT.0) Q2 =1

IF (CPT.EQ.3 .AND. F.GT.0) IPRED = LOG(F* SLOPE)

$\mathrm{Y} 2=\mathrm{IPRED}+\mathrm{EPS}(2)$

$\mathrm{Y}=\mathrm{Q} 1 * \mathrm{Y} 1+\mathrm{Q} 2 * \mathrm{Y} 2$

\section{REFERENCES}

1. Guthrie R, Susi A. A simple phenylalanine method for detecting phenylketonuria in large populations of newborn infants. Pediatrics. 1963;32:338-43.

2. Hoogtanders K, der HJ V, Christiaans M, de PA V, Van Hooff J, Stolk L. Dried blood spot measurement of tacrolimus is promising for patient monitoring. Transplantation. 2007;83(2):237-8.

3. Smit PW, Sollis KA, Fiscus S, Ford N, Vitoria M, Essajee S, et al. Systematic review of the use of dried blood spots for monitoring HIV viral load and for early infant diagnosis. PLoS ONE. 2014;9(3):e86461.

4. Beaudette P, Bateman KP. Discovery stage pharmacokinetics using dried blood spots. J Chromatogr B Analyt Technol Biomed Life Sci. 2004;809(1):153-8.

5. Emmons G, Rowland M. Pharmacokinetic considerations as to when to use dried blood spot sampling. Bioanalysis. 2010;2(11):1791-6.

6. Xu Y, Woolf EJ, Agrawal NG, Kothare P, Pucci V, Bateman KP. Merck's perspective on the implementation of dried blood spot technology in clinical drug development-why, when and how. Bioanalysis. 2013;5(3):341-50.

7. Martin NJ, Cooper HJ. Challenges and opportunities in mass spectrometric analysis of proteins from dried blood spots. Expert Rev Proteomics. 2014;11(6):685-95.

8. McDade TW, Williams S, Snodgrass JJ. What a drop can do: dried blood spots as a minimally invasive method for integrating biomarkers into population-based research. Demography. 2007;44(4):899-925.

9. Timmerman P, White S, Cobb Z, de Vries R, Thomas E, van Baar B. Update of the EBF recommendation for the use of DBS in regulated bioanalysis integrating the conclusions from the EBF DBS-microsampling consortium. Bioanalysis. 2013;5(17):2129-36.

10. Evans C, Arnold M, Bryan P, Duggan J, James CA, Li W, et al. Implementing dried blood spot sampling for clinical pharmacokinetic determinations: considerations from the IQ Consortium Microsampling Working Group. AAPS J. 2015;17(2):292-300.

11. Rowland $M$, Shepard $T$. Interpretation of microsampling data during drug development and regulatory considerations. In: Zane P, Emmons G, editors. Microsampling in pharmaceutical bioanalysis.London: Future Science Ltd; 2014. p. 121-33.

12. Rowland M, Emmons GT. Use of dried blood spots in drug development: pharmacokinetic considerations. AAPS J. 2010;12(3):290-3.

13. Xu Y, Fang W, Zeng W, Leijen S, Woolf EJ. Evaluation of dried blood spot (DBS) technology versus plasma analysis for the determination of MK-1775 by HILIC-MS/MS in support of clinical studies. Anal Bioanal Chem. 2012;404(10):3037-48. 